\title{
Adaptive Radial Basis Function Methods for Time Dependent Partial Differential Equations
}

\author{
Scott A. Sarra \\ Department of Mathematics, Marshall University, One John Marshall Drive, \\ Huntington, $W V, 25755-2560$.
}

\begin{abstract}
Radial basis function (RBF) methods have shown the potential to be a universal grid free method for the numerical solution of partial differential equations. Both global and compactly supported basis functions may be used in the methods to achieve a higher order of accuracy. In this paper, we take advantage of the grid free property of the methods and use an adaptive algorithm to choose the location of the collocation points. The RBF methods produce results similar to the more well known and analyzed spectral methods, but while allowing greater flexibility in the choice of grid point locations. The adaptive RBF methods are most successful when the basis functions are chosen so that the PDE solution can be approximated well with a small number of the basis functions.
\end{abstract}

\section{Introduction}

Ultimately, we are interested in adaptive radial basis function (RBF) PDE algorithms in two and three spatial dimensions. In this paper, we gain insight in one dimension before proceeding to higher dimensions. The implementation and complexity of RBF methods in higher dimensions are essentially the same as in one dimension. Only the adaptive algorithm will need to be different.

RBF methods for time dependent PDEs enjoy large advantages in accuracy over other flexible, but low order methods, such finite differences, finite volumes, and finite elements. However, RBF methods share the ease of implementation and flexibility of these lower order methods. Moving grid RBF methods

Email address: scott@scottsarra.org (Scott A. Sarra).

URL: www.scottsarra.org (Scott A. Sarra). 
are easily implemented, potentially even in complex computational domains in several space dimensions. Other highly accurate spatial discretization schemes such as pseudospectral methods do not have the inherent flexibility of the $\mathrm{RBF}$ methods and adaptation and complex geometries are more difficult to deal with. We have applied a modification of a simple moving grid algorithm, which was developed for use with low order finite difference methods, to RBF methods for time dependent PDEs. The adaptive RBF algorithm produces excellent results.

The numerical solution of PDEs by RBF methods is based on a scattered data interpolation problem which we review in this section. Let $x_{0}, x_{1}, \ldots, x_{N} \in$ $\Omega \subset \mathcal{R}^{n}$ be a given set of centers. A radial basis function is a function $\phi_{i}(x)=$ $\phi\left(\left\|x-x_{i}\right\|_{2}\right)$, which depends only on the distance between $x \in \mathcal{R}^{d}$ and a fixed point $x_{j} \in \mathcal{R}^{d}$. Each function $\phi_{j}$ is radially symmetric about the center $x_{j}$. The radial basis function interpolation problem may be described as, given data $f_{i}=f\left(x_{i}\right), i=0,1, \ldots, N$, the interpolating $\mathrm{RBF}$ approximation is

$$
s(x)=\sum_{i=0}^{N} \lambda_{i} \phi_{i}(x)
$$

where the expansion coefficients, $\lambda_{i}$, are chosen so that $s\left(x_{i}\right)=f_{i}$. That is, they are obtained by solving the linear system

$$
H \lambda=f
$$

where the elements of the interpolation matrix are $H_{i, j}=\phi\left(\left\|x_{i}-x_{j}\right\|_{2}\right), \lambda=$ $\left[\lambda_{0}, \ldots, \lambda_{N}\right]^{T}$, and $f=\left[f_{0}, \ldots, f_{N}\right]^{T}$. For the RBFs that we have considered in this work (table 1 and equation (8)), the interpolation matrix can be shown to be invertible for distinct interpolation points $[21,26]$.

A generalized interpolation problem also may be considered. The generalized interpolation problem is

$$
s(x)=\sum_{i=0}^{N} \lambda_{i} \phi_{i}(x)+\sum_{k=1}^{M} b_{k} p_{k}(x)
$$

in which a finite number of $d$-variate polynomials of at most order $M$ are added to the RBF basis. The polynomials $p_{k}(x)$ are the polynomials spanning $\pi_{M}$, that is they are the polynomials of degree at most $M$. The extra equation(s) needed to complete the generalized interpolation problem are chosen to be

$$
\sum_{j=0}^{N} \lambda_{j} p_{k}\left(x_{j}\right)=0
$$


for $k=1, \ldots, M$. Interpolation problem (3) must be considered when using RBFs, such as the cubics $\phi(r)=r^{3}$, as the basic interpolation problem (1) does not lead to a guaranteed invertible interpolation matrix [22]. Also, the generalized interpolation problem may lead to an approximation with some desirable properties that an approximation from the standard interpolation problem may lack, such as a degree of polynomial accuracy. This is the case with the multiquadric RBF [13].

Despite the fact that $H$ can be shown to be invertible for all $\phi$ of the interest, the linear system (2) may often be very ill-conditioned and it may be impossible to solve accurately using standard floating point arithmetic. The conditioning of $H$ is measured by the condition number defined as

$$
\kappa(H)=\|H\|\left\|H^{-1}\right\|=\sigma_{\max } / \sigma_{\min }
$$

where $\sigma$ are the singular values of $H$. The condition number of $H$ is influenced by the number of centers, the minimum separation distance of the centers, as well as values of parameters, defined below, such as the shape parameter and the support.

\section{Radial Basis Functions}

The choice of basis function is another of the flexible features of RBF methods. We will review some properties of the RBFs that we use in the numerical examples. RBFs can be globally supported, infinitely differentiable, and contain a free parameter, $\epsilon$, called the shape parameter. Representatives of this type of RBF are listed in table 1 . The global nature of RBFs of this type leads to a dense interpolation matrix. Global, infinitely differentiable RBFs typically interpolate smooth data with spectral accuracy. Details can be found in the references $[5,6,19,20]$.

The shape parameter affects both the accuracy of the approximation and the conditioning of the interpolation matrix. In general, for a fixed number of centers $N$, smaller shape parameters produce the more accurate approximations, but also are associated with a poorly conditioned $H$. The condition number also grows with $N$ for fixed values of the shape parameter $\epsilon$. In practice, the shape parameter must be adjusted with the number of centers in order to produce a interpolation matrix which is well conditioned enough to be inverted in finite precision arithmetic. Many researchers (e.g. [8,23]) have attempted to develop algorithms for selecting optimal values of the shape parameter. By optimal, we mean the value of the shape parameter that produces the most accurate interpolant. However, results in this area have been limited by the realities of floating point arithmetic. The optimal choice of the shape parameter 
is still an open question. In practice it is most often selected by brute force. Recently, Fornberg et. al. [12] developed a Contour-Padé algorithm which is capable of stably computing the RBF approximation for all $\epsilon \geq 0$. The results of using the Contour-Padé algorithm have shown that the optimal value of the shape parameter may not be reachable in standard floating point precision when applying traditional algorithms such as Gaussian elimination to solve the system (2). Several different strategies [17] have been somewhat successful in reducing the ill-conditioning problem when using RBF methods in PDE problems. The strategies include: variable shape parameters, domain decomposition, preconditioning the interpolation matrix, and optimizing the center locations. Often, more than one of these strategies are used together.

In our numerical examples, we have used the multiquadric (MQ) RBF which is defined in table 1. Alternatively, the MQ may be defined as $\phi(r, c)=\sqrt{r^{2}+c^{2}}$. This is seen to be equivalent to our definition with $\epsilon=1 / c$. It seems more natural to define the MQ in this way rather than in the traditional way, as the shape parameter now behaves in the same way it does in other infinitely smooth RBFs. The behavior as $\epsilon \rightarrow 0$ is that the interpolant becomes more accurate, the condition number of the interpolation matrix gets larger, and the shape of the RBF becomes flatter.

For approximation with the MQ RBF we consider the generalized interpolation problem (3) with $M=1$. The interpolation problem with $M=1$ takes the form

$$
s(x)=\sum_{i=0}^{N} \lambda_{i} \phi_{i}(x)+b
$$

where $b$ is a constant and the auxiliary equation is

$$
\sum_{i=0}^{N} \lambda_{i}=0 .
$$

The resulting interpolation matrix will be of the form

$$
H=\left[\begin{array}{cccc}
\phi\left(\left\|x_{0}-x_{0}\right\|_{2}\right) & \cdots & \phi\left(\left\|x_{0}-x_{N}\right\|_{2}\right) & 1 \\
\vdots & \ddots & \vdots & \vdots \\
\phi\left(\left\|x_{N}-x_{0}\right\|_{2}\right) & \cdots & \phi\left(\left\|x_{N}-x_{N}\right\|_{2}\right) & 1 \\
1 & \cdots & 1 & 0
\end{array}\right]
$$

For the MQ the interpolation matrix constructed from the generalized interpolation problem with $M=1$ is guaranteed to be invertible for distinct centers 


\begin{tabular}{|l|l|}
\hline Name of RBF & Definition \\
\hline Multiquadric (MQ) & $\phi(r, \epsilon)=\sqrt{1+(\epsilon r)^{2}}$ \\
Inverse Quadratics (IQ) & $\phi(r, \epsilon)=1 /\left(1+(\epsilon r)^{2}\right)$ \\
Inverse Multiquadric (IMQ) & $\phi(r, \epsilon)=1 / \sqrt{1+(\epsilon r)^{2}}$ \\
Gaussian (GA) & $\phi(r, \epsilon)=e^{-(\epsilon r)^{2}}$ \\
\hline
\end{tabular}

Table 1

Global, infinitely smooth RBFs

[22]. For a discussion of the merits of using the MQ RBF with an appended constant see references [13] and [22].

An alternative to the global, infinitely smooth RBFs are compactly supported RBFs (CSRBFs). Wendland's CSRBFs [26] are representative of this class. The Wendland functions, $\phi_{\ell, \kappa}$, are strictly positive definite in $\mathcal{R}^{d}$ for all $d \leq d_{0}$ and can be constructed to have any desired amount of smoothness $2 \kappa$, i.e., $\phi \in C^{2 \kappa}$. The parameter $\ell$ is $\ell=\left\lfloor\frac{d}{2}\right\rfloor+\kappa+1$. For $\kappa=0,1,2,3$, the functions can be computed by an explicit formulae [10]. The Wendland functions are defined to have compact support on the interval $[0,1]$ but may be scaled to have compact support on $[0, \delta]$ by replacing $r$ with $r / \delta$ for $\delta>0$. The scaling factor $\delta$ can be constant or it can be variable at different centers. A way to specify the optimal value of $\delta$ is currently not known. In our numerical experiments we have used the Wendland CSRBF

$$
\phi_{4,2}=(1-r)_{+}^{6}\left(3+18 r+35 r^{2}\right)
$$

where

$$
(1-r)_{+}^{6}= \begin{cases}(1-r)_{+}^{6} & 0 \leq r<1 \\ 0 & r \geq 1\end{cases}
$$

which are in $C^{4}$ and are positive definite in up to three space dimensions. Since $\phi_{4,2}(\mathrm{~W} 42)$ is positive definite we need only consider the standard interpolation problem (1) and the matrix $H$ will be nonsingular for a distinct set of centers. If the support of the basis functions are small compared to the size of the computational domain of the PDE, banded matrix algorithms can be used to invert the interpolation matrix. Error estimates [27] for approximations of $f \in H^{s}\left(\mathcal{R}^{d}\right)$ by Wendland's CSRBFs are of the form

$$
\left\|f-s_{f}\right\|_{L_{\infty}(\Omega)} \leq C h^{k+\frac{1}{2}}\|f\|_{H^{s}\left(\mathcal{R}^{d}\right)}
$$

where h denotes the "meshsize", i.e., the separation distance of the centers, $h=\sup _{x \in \Omega} \min \left\|x-x_{j}\right\|$ for $x_{j} \in \mathcal{R}^{d} . H^{s}\left(\mathcal{R}^{d}\right)$ is the usual Sobolev space 
of functions with $s$ derivatives bounded in $L^{2}$ and $s=\frac{d}{2}+k+\frac{1}{2}$ gives the regularity of the data.

\section{RBF methods for time dependent PDEs}

Derivatives of the interpolant (1) or (3) may be calculated in a straightforward manner. For instance, using the interpolant (1) in $\mathcal{R}$, the derivatives at the centers $x_{j}$ can be calculated as

$$
s^{(n)}\left(x_{j}\right)=\sum_{i=0}^{N} \lambda_{i} \phi_{i}^{(n)}\left(x_{j}\right) .
$$

for $n=1,2, \ldots$. The spatial derivatives can be written compactly in matrix form as

$$
s^{(n)}=H^{(n)} \lambda
$$

where the elements of $H^{(n)}$ are $\phi^{(n)}\left(\left\|x_{i}-x_{j}\right\|_{2}\right)$.

In the context of a time dependent PDE method, where derivatives may need to be evaluated thousands of times, it is often more efficient to form the derivative matrix

$$
D^{(n)}=H^{(n)} H^{-1}
$$

Then spatial derivatives can be approximated by a single matrix by vector multiplication

$$
s^{(n)}=D^{(n)} s .
$$

To describe how to implement a RBF method for solving a time dependent PDE on a fixed grid, we use Burgers' equation (23) as an example. A fixed time step has been used, but variable time stepping is possible. The PDE is discretized in space with radial basis functions to get the semi-discrete system

$$
s_{t}=F(s) .
$$

The system of ODEs (15) is then advanced in time with any ODE method. In the numerical examples, we have used an explicit fourth-order Runge-Kutta method. At time $t=0$ the derivative matrixes, $D^{(1)}$ and $D^{(2)}$, are constructed. 
At each internal Runge-Kutta stage, we calculate $s^{(1)}=D^{(1)} s, s^{(2)}=D^{(2)} s$, and then for $i=0, \ldots, N, F_{i}=\nu s^{(2)}\left(x_{i}\right)-s\left(x_{i}\right) s^{(1)}\left(x_{i}\right)$. The implementation of the method is extremely simple.

\section{Adaptive Grids}

It has generally been accepted, at least for problems in one space dimension, that adaptive grid methods are capable of resolving PDE solutions that contain regions of rapid variation with acceptable accuracy and without using an excessive number of grid points. Adaptive grid methods and applications in one space dimension, have been extensively studied. Many one-dimensional adaptive grid algorithms for time-dependent PDEs in the context of finite difference, finite element, and pseudospectral methods have been described. Details and further references may be found in $[1,2,4,14-16]$. The adaptive grid algorithm that we have used is a slightly modification of the equidistribution of arclength algorithm for one dimensional systems of PDEs described in [24]. We have modified the interpolation step, which used cubic polynomials at interior nodes and quadratic polynomials at the nodes next to the boundary, to instead use the same RBFs used in the PDE solution at all nodes. Thus, the method does not require any modifications near the boundaries.

This allows the adaptive RBF methods to maintain an overall high order of accuracy. When we apply the adaptive algorithm with second-order finite differences, we have retained the cubic interpolation step. The algorithm is simple and computationally inexpensive in that it is not necessary to transform the original PDE into a new coordinate system, or is it necessary to solve an additional companion PDE to choose the coordinate system and node distribution. Other algorithms may result in different, and possibly "better" grids being used, but for our purposes, the features of the RBF methods we wish to examine will remain very similar, regardless of particular adaptive algorithm used.

In the adaptive algorithm, we start at time $t^{0}$ with a uniform grid $x_{j}^{0}$. To advance the PDE in time with the adaptive grid algorithm, we start by assuming that at time level $t^{n}$ we have computed approximate solutions $s_{j}^{n}$, by a radial basis function method, to the true solution $u\left(x_{j}^{n}, t^{n}\right)$ on a grid $x_{j}^{n}$, where $j=0, \ldots, N$. Then, the RBF method is used on the grid $x_{j}^{n}$ to obtain approximations $\bar{s}_{j}^{n+1}$ to $u\left(x_{j}^{n}, t^{n+1}\right)$. Next, the points $\left(x_{j}^{n}, \bar{s}_{j}^{n+1}\right)$ are joined by straight lines and the length $\theta^{n+1}$ of the resulting polygon is computed. Then the points $P_{j}^{n+1}$ on the polygon are found which divide its total length into $N$ equal parts. The new nodes $x_{j}^{n+1}$ are found as the projection of $P_{j}^{n+1}$ onto the $x$-axis. Finally, $s_{j}^{n+1}$, the approximation to $u\left(x_{j}^{n+1}, t^{n+1}\right)$, is computed by using RBFs to interpolate the values $\left(x_{j}^{n}, \bar{s}_{j}^{n+1}\right)$ to $\left(x_{j}^{n+1}, s_{j}^{n+1}\right)$. 
The adaptive algorithm contains two parameters that control the adaptation. The parameter $\mu$ causes the adaptation to be performed every $\mu$ time steps. The parameter $\beta$ controls the relative size of the largest and smallest grid spacings by ensuring that

$$
\max _{i} h_{i} \leq \sqrt{1+\beta} \min _{i} h_{i}
$$

where $h_{i}=x_{i}-x_{i-1}$. When using the adaptive algorithm with CSRBFs, the bandwidth, $b$, of the interpolation matrix $H$ will be

$$
b=\left\lfloor\frac{\delta}{\min _{i} h_{i}}\right\rfloor .
$$

The computational cost of choosing a new grid is relatively small. However, setting up the RBF method on the new grid involves constructing derivative matrices for the new grid. Thus, the setup costs of the adaptive method may be prohibitive, unless the PDE solution can be approximated with a relatively small number of centers or unless CSRBFs are used which lead to a narrowly banded interpolation matrix that can be efficiently inverted.

To obtain an accurate numerical approximation with RBFs and the adaptive grid algorithm, we have found that a good strategy is to monitor the condition number of the interpolation matrix $H$ that is used to form new derivative matrices and to interpolate to the new set of centers each time the solution is re-gridded. The condition number of $H$ should not exceed $5 \times 10^{10}$ during any stage of the time evolution of the solution. If the condition number does become too large, the stage should be rejected and recalculated with a smaller value of $\beta$, which decreases the minimum separation distance of the centers. Another option to reduce the condition number of $H$ for a rejected stage is to decrease the number of centers used. Both the values of $\beta$ and $N$ may be adapted when a re-gridding takes place. If it is not possible to achieve small enough condition numbers in a single domain, domain decomposition will be necessary.

\section{$5 \quad$ Numerical Results}

First, we have applied the adaptive algorithm described in section 4 to select a computational grid for a single derivative calculation. Then we have applied it to two PDE problems with solutions containing regions of rapid variation. The results are compared with pseudospectral and finite difference approximations.

The simple adaptive algorithm can be used in RBF methods to achieve an 
accuracy goal, but with significantly fewer centers than a fixed grid method would require. In order to get a point of reference for the accuracy of RBF function methods on fixed grids, we compare the accuracy of the RBF function methods on fixed grids with the well known Chebyshev pseudospectral (CPS) method [7]. Additionally, we compare the RBF methods with a centered second order finite difference method (FD2) on both fixed and adaptive grids. We illustrate the fact that the simple adaptive algorithm that we have applied in the RBF and FD2 methods is not able to be applied in CPS method since the method is restricted to a fixed grid or to mappings of that grid. This feature of the CPS method adds to the complexity of CPS adaptive algorithms and limits the possible positioning of grid points.

For all the PDE methods we have taken small uniform time steps with a fourthorder explicit Runge-Kutta method in order to make the temporal errors small. In this way, we have isolated the effects of the spatial approximations as much as possible. However, algorithms which adaptively adjust the time step could be used in conjunction with the adaptive spatial schemes to reduce the number of time steps taken.

Two types of errors were measured, the max error

$$
E^{\infty}=\max _{0 \leq i \leq N}\left|f\left(x_{i}\right)-s\left(x_{i}\right)\right|
$$

where $f(x)$ is the exact value and $s(x)$ is the RBF approximation, and the rms error

$$
E^{2}=\sqrt{\frac{1}{N} \sum_{0 \leq i \leq N}\left|f\left(x_{i}\right)-s\left(x_{i}\right)\right|^{2}} .
$$

\subsection{Single Derivative}

Our first numerical experiment compares the accuracy of the RBF methods and the CPS method for calculating a single derivative of a function with a region of rapid variation. The comparison is made on both fixed base grids and on grids adapted based on qualities on the function being approximated. The function being differentiated is the exact solution to Burgers' equation (23) from our second numerical example. We have used the solution at time $t=1.1$ with $\nu=0.01$. In both the base grid and adapted grid calculations we have used 50 grid points. RBF methods are not tied to a fixed grid, but we take an evenly spaced grid as the "base" grid of the methods. We can not do the same for the CPS method since methods that are based on high order global polynomial interpolation are unstable on uniform grids. This situation 


\begin{tabular}{|c|c|}
\hline method & $E^{2}$ error \\
\hline MQ base & 0.056371 \\
MQ adaptive & 0.000087 \\
CPS base & 0.319269 \\
$C P S_{\alpha}=0.99$ & 0.109765 \\
CPS adaptive & 0.000093 \\
\hline
\end{tabular}

Single derivative results, $N=49$

is often described by the term Runge phenomenon. Among several choices, the base grid for the CPS method is usually chosen to be

$$
x_{j}=-\cos \left(\frac{\pi j}{N}\right), \quad j=0,1, \ldots, N .
$$

The grid clusters nodes quadratically around the boundaries. The results of the base grid calculations are listed in table 2. The lack of resolution in center of the domain caused by the boundary clustering of nodes leads to a large error in the CPS method. Often, the grid (20) is redistributed via a mapping of this grid in order to lessen stable explicit time stepping limits in a time dependent PDE method or to provide greater resolution in regions other than near the boundaries. Perhaps the most used map is [18]

$$
x=\frac{\arcsin (\alpha \xi)}{\arcsin (\alpha)}
$$

which can produce a nearly evenly distributed set of grid points as $\alpha \rightarrow 1$, but is singular for $\alpha=1$. The results of using this map with $\alpha=0.99$ are listed in the $C P S_{\alpha=0.99}$ row of table 2 . The results are better than the CPS base grid results, but not as good as the MQ RBF results on the uniform grid. For the MQ RBF, the shape parameter was selected as $\epsilon=15$. A remarkable fact about radial basis functions is that they can produce spectrally accurate results to non-periodic problems on a uniform grid.

The simple adaptive algorithm that we have described in section 4 is applicable to both the RBF and FD2 methods, but can not be applied in the framework of the CPS method since the CPS method must be applied on its base grid (20), or on mapping of that grid. The simple adaptive algorithm does not account for this restriction, and more complex grid adaptation algorithms must be used for the CPS method.

Even though the adaptive algorithm we have used in the FD and RBF methods can not be used in the CPS method, adaptive algorithms have been developed 
for the CPS method. The adaptive CPS methods typically write the PDE in a transformed variable $\xi$ through a mapping $x=f\left(\xi, p_{1}, p_{2}\right)$ where $p_{1}$ is a parameter that describes the location of the rapid variation and $p_{2}$ is a parameter that controls the magnitude of the coordinate contraction near $x=p_{1}$. The values of $p_{1}$ and $p_{2}$, and thus the grid point locations, are selected by minimizing a functional of the solution that is connected with the approximation error. The functional is typically taken to be the norm of a weighted Sobolev space such as $H_{w}^{2}$, but other choices are possible [3]. The possible grids are limited to mappings of the grid (20). This grid dependence makes the CPS adaptive algorithms difficult to apply to problems with multiple regions of rapid variation and hinders extension to higher space dimensions. Although the mapping used may redistribute the grid points (20) to the interior of the domain and to regions of rapid variation, some boundary clustering will still be present. Additionally, the CPS adaptive algorithms require a multidimensional optimization step which adds to the complexity of the methods. The full description of the adaptive CPS algorithm for PDEs is beyond the scope of this work and the interested reader is referred to [2] for details and for an application of the adaptive CPS methods to Burgers' equation. Here we only apply to method to a single derivative calculation. A map that is commonly used in the CPS adaptive algorithms is

$$
x=f\left(\xi, p_{1}, p_{2}\right)=p_{1}+p_{2} \tan \left[\omega\left(\xi-\xi_{0}\right)\right]
$$

where $\xi_{0}=(\kappa-1) /(\kappa+1), \kappa=\tan ^{-1}\left(\left[1+p_{1}\right] / p_{2}\right) / \tan ^{-1}\left(\left[1-p_{1}\right] / p_{2}\right)$, and $\omega=\tan ^{-1}\left(\left[1-p_{1}\right] / p_{2}\right) /\left(1-\xi_{0}\right)$. The adaptive grid results are given in table 2. The MQ RBF grid was produced by the algorithm described in section 4 . The shape parameter used was $\epsilon=15$ and the grid adaption parameter was $\beta=18$. The CPS adaptive grid was produced by minimizing a functional based on the $H_{w}^{2}$ norm to select the parameters $p_{1}$ and $p_{2}$ in map (22). The method selects the parameters as $p_{1} \approx 0$ and $p_{2} \approx 5.3$. The base and adapted grids for the single derivative test are shown in figure 1.

The results of this simple test of calculating a single derivative illustrate several features of RBF methods. One is that they can be as accurate as spectral methods. RBF methods are grid free which allows center locations to be selected without restriction. Additionally, and important feature of RBF methods that is sometimes overlooked is that they can produce spectral accuracy on a uniform grid. 


\begin{tabular}{|c|c|c|c|c|c|}
\hline method & $\mathrm{N}$ & $E^{2}$ & $E^{\infty}$ & supp & $\epsilon$ \\
\hline$M Q$ & 103 & 0.00196 & 0.0092 & - & 11 \\
$\mathrm{~W} 42$ & 103 & 0.00159 & 0.0129 & 2 & - \\
$\mathrm{W} 42$ & 103 & 0.00183 & 0.0159 & 0.6 & - \\
$\mathrm{FD} 2$ & 340 & 0.00198 & 0.01889 & - & - \\
$C P S_{\alpha=0.99}$ & 113 & 0.00181 & 0.0068 & - & - \\
$M Q_{\alpha=0.99}$ & 113 & 0.00173 & 0.0082 & - & 10 \\
$W 42_{\alpha=0.99}$ & 111 & 0.00172 & 0.0144 & 2 & - \\
\hline
\end{tabular}

Table 3

Fixed grid Burgers' results, $\nu=0.0035$.

\subsection{Burgers' Equation}

Our first PDE problem is Burgers' equation

$$
u_{t}+u u_{x}=\nu u_{x x}
$$

on the interval $[-1,1]$. The exact solution to the test problem is

$$
u(x, t)=\frac{0.1 e^{a}+0.5 e^{b}+e^{c}}{e^{a}+e^{b}+e^{c}} .
$$

where $a=-(x+0.5+4.95 t) /(20 \nu), b=-(x+0.5+0.75 t) /(4 \nu)$, and $c=$ $-(x+0.625) /(2 \nu)$. The initial condition, $u(x, 0)$, and the boundary conditions $u(-1, t)=g_{l}(t)$ and $u(1, t)=g_{r}(t)$ are specified using the exact solution. We have taken $\nu=0.0035$ which makes the viscous term small and allows a steep front to develop in the solution. The exact solution is illustrated in the left image of figure 2. In our numerical results we seek an $E^{2}$ error less than 0.002 at time $t=1.1$. At this time the steep front has completely developed. The time step used was $\Delta t=0.002$. Fixed grid results for Burgers' equation are in table 3. Non-subscripted results are on uniformly spaced grids, while methods with subscripts of $\alpha$ indicate that the fixed, but non-uniformly spaced grid has been formed using equation (21). For the CPS method, we used a grid formed with (21) with $\alpha=0.99$ to get a grid with spacing closer to uniform than the base grid (20). Without adaptation, both the global MQ and compactly supported W42 RBF methods perform better than the CPS method on this problem. The W42 RBF does this with support ranging from supp $=0.6$ to a full support of supp $=2.0$. The uniform distribution of grid points in the RBF methods allow better interior resolution of the steep front in the center of the domain. In problems lacking inherent dissipative mechanisms such as our next example, the advection equation, the clustering of centers near the boundary 


\begin{tabular}{|c|c|c|c|c|c|c|c|}
\hline method & $\mathrm{N}$ & $\beta$ & $\mu$ & $E^{2}$ & $E^{\infty}$ & supp & $\epsilon$ \\
\hline$M Q$ & 47 & 35 & 12 & 0.00191 & 0.00947 & - & 52 \\
$\mathrm{~W} 42$ & 60 & 30 & 10 & 0.00175 & 0.00670 & 2 & - \\
$\mathrm{W} 42$ & 60 & 30 & 10 & 0.00129 & 0.00489 & 1.25 & - \\
\hline
\end{tabular}

Table 4

Adaptive Burgers' results, $\nu=0.0035$.

can reduce errors in the $\mathrm{RBF}$ methods that occur in the boundary regions. However, in problems such as Burgers' equation with build in dissipation, there does not seem to be any benefit to clustering centers around the boundary, unless regions of rapid variation are near the boundary.

The adaptive grid Burgers' results are shown in table 4. Using the MQ RBF method, the adaptive algorithm was able to meet the accuracy goal using less than half as many centers as the fixed grid methods. The solution at $t=1.1$ using the adaptive MQ algorithm is shown in figure 3 . The condition numbers of the interpolation matrix that must be inverted each time a new grid is chosen ranged from $8.4 \times 10^{3}$ to $1.7 \times 10^{6}$ for the MQ RBFs and $2 \times 10^{7}$ to $4.6 \times 10^{10}$ for the W42 RBFs. Throughout the time stepping, the matrix condition numbers were monitored to ensure that they did not exceed $5.0 \times 10^{10}$.

It is interesting to note that the adaptive FD2 algorithm was not able to achieve the accuracy goal with any number of grid points. It is suspected that the third order interpolation stage was not capable of producing a sharp enough edge at the steep front. The FD2 adaptive solution was smeared in this area.

Finally, we consider an extreme version of the previous test problem in which we use only a fourth as much viscosity, $\nu=0.000875$. The solutions to the problems are nearly piecewise constant as is illustrated in the right image of figure 2. The fixed grid results are shown in table 5. Without adaptation, the Chebyshev pseudospectral method needs a large number of nodes due to Gibbs-like oscillations around the steep fronts unless a spectral filter [25] is applied. The large number of grid points used by the CPS method also required that a very small time step be used for stability in the explicit RK4 method. The RBF methods perform better than the CPS method without adaptation and significantly better than the finite difference method. For the adaptive RBF method, several combinations of $\beta, \mu$, supp, and $\epsilon$ resulted in the accuracy goal being met, two of which are listed in table 6 . With such steep fronts to be resolved, it is difficult to place enough centers in the vicinity of the fronts without the interpolation matrix becoming very poorly conditioned. This necessitated a very large shape parameter being used in the adaptive MQ method. Using the MQ method with a large shape parameter often results 


\begin{tabular}{|c|c|c|c|c|c|c|}
\hline method & $\mathrm{N}$ & $E^{2}$ & $E^{\infty}$ & supp & $\epsilon$ & $\Delta t$ \\
\hline$M Q$ & 299 & 0.00179 & 0.01543 & - & 21 & 0.001 \\
$\mathrm{~W} 42$ & 339 & 0.00191 & 0.02696 & 0.8 & - & 0.002 \\
$\mathrm{FD} 2$ & 1000 & 0.00193 & 0.0369 & - & - & 0.0005 \\
$C P S_{\alpha=0.99}$ & 429 & 0.00157 & 0.0206 & - & - & 0.000025 \\
\hline
\end{tabular}

Table 5

Fixed grid Burgers' results, $\nu=0.000875$,

\begin{tabular}{|c|c|c|c|c|c|c|c|c|}
\hline method & $\mathrm{N}$ & $\beta$ & $\mu$ & $E^{2}$ & $E^{\infty}$ & supp & $\epsilon$ & $\Delta t$ \\
\hline$M Q$ & 139 & 100 & 20 & 0.00196 & 0.007455 & - & 110 & 0.0005 \\
$\mathrm{~W} 42$ & 149 & 20 & 10 & 0.00177 & 0.00938 & 0.8 & - & 0.002 \\
\hline
\end{tabular}

Table 6

Adaptive Burgers' results, $\nu=0.000875$.

in accuracy comparable to lower order finite difference methods. However, despite the large shape parameter, the MQ method produced good results while the adaptive FD2 algorithm was unable to meet the accuracy goal with any number of grid points.

\subsection{Advection Equation}

Our next numerical experiment is with the advection equation

$$
u_{t}+u_{x}=0
$$

with initial condition $u(x, 0)=e^{-2000(x+1)^{2}}$ and boundary condition $u(-1, t)=$ $g(t)=e^{-2000 t^{2}}$. In our numerical results we seek an $E^{2}$ error less than 0.002 at time $t=1.0$ when the thin pulse has moved to the center of the domain. Table 7 gives results on fixed grids. A small time step of $\Delta t=0.001$ was used. Non-subscripted results are on uniformly spaced grids, while methods with subscripts of $\alpha$ indicate that the fixed, but non-uniformly spaced grid has been formed using equation (21). It is well known that the largest errors in RBF methods occur near boundaries [11], especially in non-dissipative wave type problems such as the advection equation. The reduction of boundary errors in this type of problem has become an active area of research. One possible way to lessen the boundary errors is to cluster centers around the boundaries as pseudospectral methods do. The results of the boundary clustering of centers can be seen in the $M Q_{\alpha=0.99}$ and $M Q_{\alpha=0.9975}$ results. With a properly chosen grid, both the CPS and the MQ RBF method can meet the accuracy goal with $N=111$. As expected, the second order finite difference method was not competitive with the RBF or CPS methods. 


\begin{tabular}{|c|c|c|c|c|c|}
\hline method & $\mathrm{N}$ & $E^{2}$ & $E^{\infty}$ & supp & $\epsilon$ \\
\hline$M Q$ & 169 & 0.00193 & 0.01825 & - & 24 \\
$\mathrm{~W} 42$ & 267 & 0.00195 & 0.01934 & 2 & - \\
$\mathrm{W} 42$ & 229 & 0.00192 & 0.01818 & 0.1 & - \\
$\mathrm{FD} 2$ & 3700 & 0.00199 & 0.01716 & - & - \\
$C P S_{\alpha=0.99}$ & 111 & 0.00178 & 0.00621 & - & - \\
$M Q_{\alpha=0.99}$ & 125 & 0.00189 & 0.00721 & - & 8.5 \\
$M Q_{\alpha=0.9975}$ & 111 & 0.00167 & 0.00198 & - & 6.4 \\
\hline
\end{tabular}

Table 7

Fixed grid Advection results

\begin{tabular}{|c|c|c|c|c|c|c|c|}
\hline method & $\mathrm{N}$ & $\beta$ & $\mu$ & $E^{2}$ & $E^{\infty}$ & supp & $\epsilon$ \\
\hline$M Q$ & 61 & 48 & 2 & 0.00197 & 0.00805 & - & 31 \\
$\mathrm{~W} 42$ & 49 & 500 & 2 & 0.00141 & 0.00395 & 0.07 & - \\
FD2 $^{*}$ & 1250 & 150 & 10 & 0.00150 & 0.00925 & - & - \\
\hline
\end{tabular}

Table 8

Adaptive Advection results

Table 8 gives the adaptive grid results. A small time step of $\Delta t=0.001$ was used except for the FD2, which required a smaller time step for stability and $\Delta t=0.00025$ was used. With the grid parameter $\beta=500$ in the W42 calculation, the largest grid spacing may be as much as 22 times larger than the smallest grid spacing. In figure 4 this wide grid spacing can be observed in the flat regions of the solution. Despite the fact that the MQ interpolant is spectrally accurate and that the W42 approximation has only a fixed algebraic convergence rate, the W42 RBF was able to attain the accuracy goal with less nodes than the MQ RBF. Heuristically, this can be explained by the fact that with a support of 0.07 the W42 RBFs have a shape similar to the features in the PDE solution, while the MQ does not mimic the features of the PDE solution as well. This is in agreement with one of the basic tenants of approximation theory - choose a basis in which a function can be well represented by a small number of basis functions.

The condition numbers of the interpolation matrix that must be inverted each time a new grid is chosen ranged from $9.5 \times 10^{7}$ to $3.4 \times 10^{9}$ for the MQ basis functions and $1.0 \times 10^{2}$ to $2.1 \times 10^{6}$ for the W42 basis functions. The structure and sparse nature of the interpolation matrix for the adaptive W42 calculation at $t=1.0$ with $\delta=0.07$ is shown in figure 5 . 


\section{Conclusions}

Radial Basis Function methods were used to solve two PDEs with solutions containing regions of rapid variation. Without the use of grid adaptation, the RBF methods were competitive in both accuracy and computational cost with the Chebyshev pseudospectral method. The inherent flexility of the RBF methods allowed the node location to be chosen adaptively in a way that retained the desired accuracy, but used significantly fewer centers. This complete freedom choice of center location is lacking in pseudospectral methods, since adaptations are limited to mappings of a fixed, non-uniformly spaced grid. The gridless feature of RBF methods will allow PDEs with solutions having multiple regions of rapid variation, and problems in higher dimensions, to be handled equally as well. The ability of the RBF methods to use a fixed uniformly space grid is another advantage of the RBF methods over the CPS method.

The choice of basis function is another flexible feature of RBF methods. Basis functions may have global or compact support and may have varying degrees of smoothness. It was found in our numerical results that the "best" choice of basis function for a particular problem was one in which the shapes of the basis functions best matched the shapes or features of the PDE solution. This allowed the solution to be approximated well with a small number of basis functions.

Our future research will be concerned with adaptive grid RBF methods in two and three space dimensions. We will be concerned with both existing adaptive algorithms and new algorithms specifically tailored to RBF methods. The close connection between RBFs and wavelets [9] will be explored with the goal of using wavelets to guide the adaptation.

\section{References}

[1] S. Adjerid and J. E. Flaherty. A moving finite element method with error estimation and refinement for one-dimensional time dependent partial differential equations. SIAM Journal on Numerical Analysis, 23:778-796, 1986.

[2] J.M. Augenbaum. An adaptive pseudospectral method for discontinuous problems. Applied Numerical Mathematics, 5:459-480, 1989.

[3] A. Bayliss, D. Gottlieb, B. J. Matkowsky, and M. Minkoff. An adaptive pseudospectral method for reaction-diffusion problems. Journal of Computational Physics, 81:421-443, 1989.

[4] G. Beckett, J. Mackenzie, A. Ramage, and D. Sloan. On the numerical solution 
of one-dimensional PDEs using adaptive methods based on equidistribution. Journal of Computational Physics, 167:372-392, 2001.

[5] M. D. Buhmann. Spectral convergence of multiquadric interpolation. Proceedings of the Edinburgh Mathematical Society, 36:319-333, 1993.

[6] Martin D. Buhmann. Radial Basis Functions. Cambridge University Press, 2003.

[7] Claudio Canuto, M. Y. Hussaini, Alfio Quarteroni, and Thomas A. Zang. Spectral Methods for Fluid Dynamics. Springer-Verlag, New York, 1988.

[8] R. E. Carlson and T. A. Foley. The parameter $r^{2}$ in multiquadric interpolation. Computers and Mathematics with Applications, 21(9):29-42, 1991.

[9] C. K. Chui, J. Stockler, and J. D. Ward. Analytic wavelets generated by radial function. Advances in computational mathematics, 5:95-123, 1996.

[10] G. E. Fasshauer. On smoothing for multilevel approximation with radial basis functions. In Charles Chui and Lary Schumaker, editors, Approximation Theory IX. Vanderbilt University Press, 1998.

[11] B. Fornberg, T. Dirscol, G. Wright, and R. Charles. Observations on the behavior of radial basis function approximations near boundaries. Computers and Mathematics with Applications, 43:473-490, 2002.

[12] B. Fornberg and G. Wright. Stable computation of multiquadric interpolants for all values of the shape parameter. To appear in Computers and Mathematics with Applications, 2004.

[13] R. L. Hardy. Theory and applications of the multiquadric-biharmonic method. Computers and Mathematics with Applications, 19(8/9):163-208, 1990.

[14] D. Hawken, J. Gottlieb, and J. Hansen. Review of some adaptive nodemovement techniques in finite element and finite difference solutions of PDEs. Journal of Computational Physics, 95:254, 1991.

[15] W. Huang and R.D. Russell. A moving collocation method for solving time dependent partial differential equations. Applied Numerical Mathematics, 20:101-116, 1996.

[16] Leland Jameson. A wavelet-optimized, very high order adaptive grid and order numerical method. SIAM Journal on Scientific Computing, 19(6):1980-2013, 1998.

[17] E. Kaansa and Y.C. Hon. Circumventing the ill-conditioning problem with multiquadric radial basis fuctions: Applications to elliptic partial differential equations. Computers and Mathematics with Applications, 39(7/8):123-137, 2000 .

[18] R. Kosloff and H. Tal-Ezer. A modified Chebyshev pseudospectral method with an $\mathrm{O}(1 / \mathrm{N})$ time step restriction. Journal of Computational Physics, 104:457$469,1993$. 
[19] W. R. Madych and S. A. Nelson. Error bounds for multiquadric interpolation. In C. Chui, L. Schumaker, and J. Ward, editors, Approximation Therory VI, pages 413-416. Academic Press, 1989.

[20] W. R. Madych and S. A. Nelson. Multivariate interpolation and conditionally positive definite functions ii. Mathematics of Computation, 4(189):211-230, 1990.

[21] C. Micchelli. Interpolation of scattered data: Distance matrices and conditionally positive definite functions. Constructive Approximation, 2:11-22, 1986.

[22] M. Powell. The theory of radial basis function approximation in 1990. In W. Light, editor, Advances in Numerical Analysis, Vol. II: Wavelets, Subdivision Algorithms and Radial Functions. 1990.

[23] S. Rippa. An algorithm for selecting a good parameter c in radial basis function interpolation. Advances in Computational Mathematics, 11:193-210, 1999.

[24] J. Sanz-Serna and I. Christie. A simple adaptive technique for nonlinear wave problems. Journal of Computational Physics, 67:348-360, 1986.

[25] H. Vandeven. Family of spectral filters for discontinuous problems. SIAM Journal of Scientific Computing, 6:159-192, 1991.

[26] H. Wendland. Piecewise polynomial, positive definite and compactly supported radial funtions of minimal degree. Advances in Compuational Mathematics, 4:389-396, 1995.

[27] H. Wendland. Error estimates for interpolation by compactly supported radial basis functions of minimal degree. Journal of Approximation Theory, 93:258$272,1998$. 


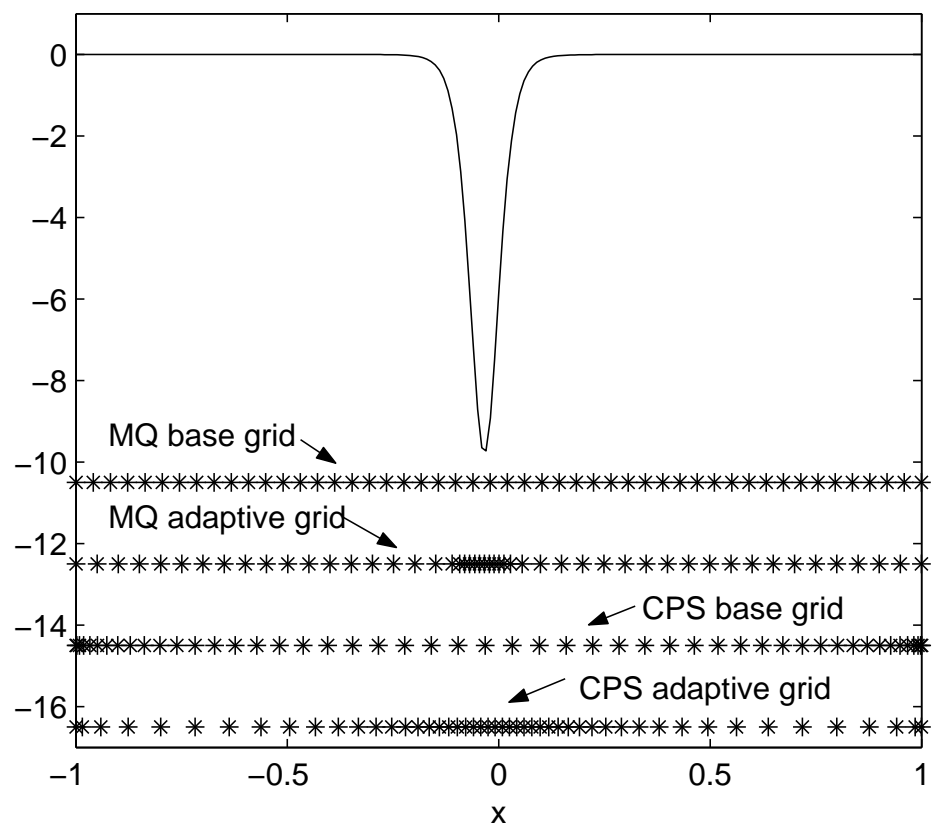

Fig. 1. Derivative of the solution of Burgers' equation (23) with $\nu=0.01$ at $t=1.1$. Also displayed are the grids from the numerical experiment of section 5.1 which approximates the derivative of the solution profile on base and adaptive grids using the MQ RBF method and the Chebyshev pseudospectral method.
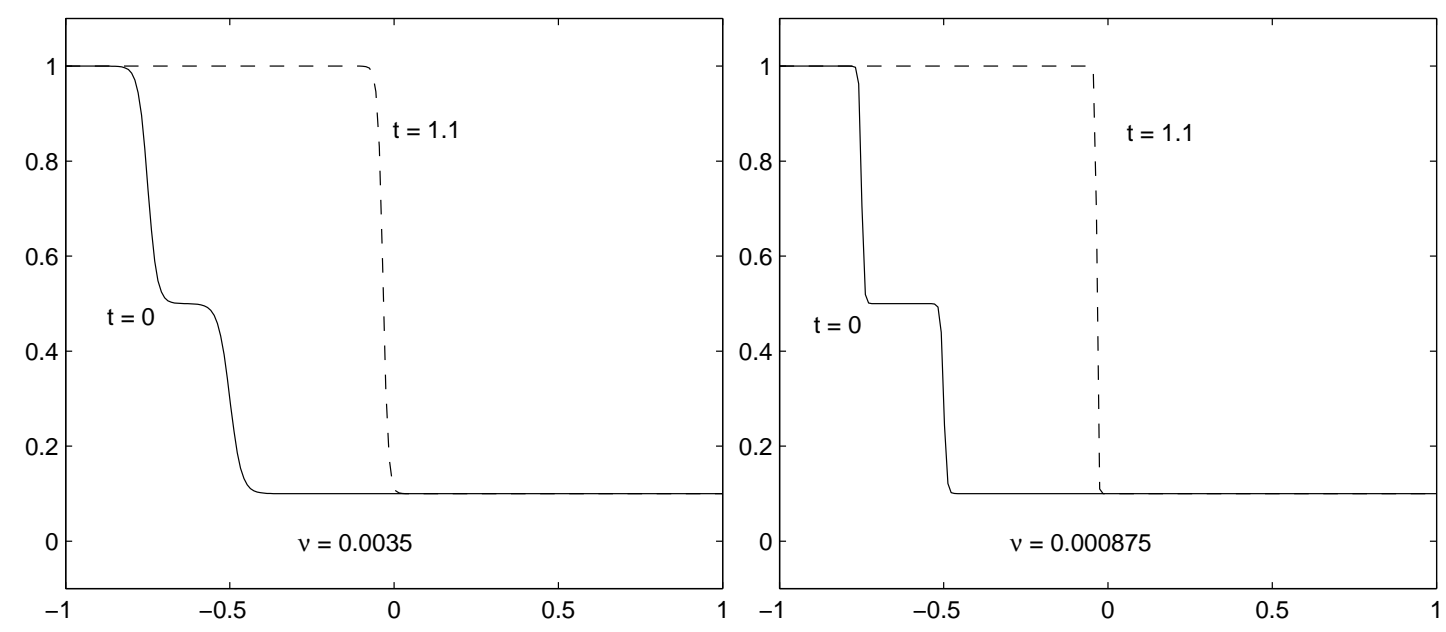

Fig. 2. Exact solution of Burgers' (23) equation (solid) from section 5.2 at $t=0$ and $t=1.1$ (dashed). Left: $\nu=0.0035$. Right $\nu=8.75 e-4$. 


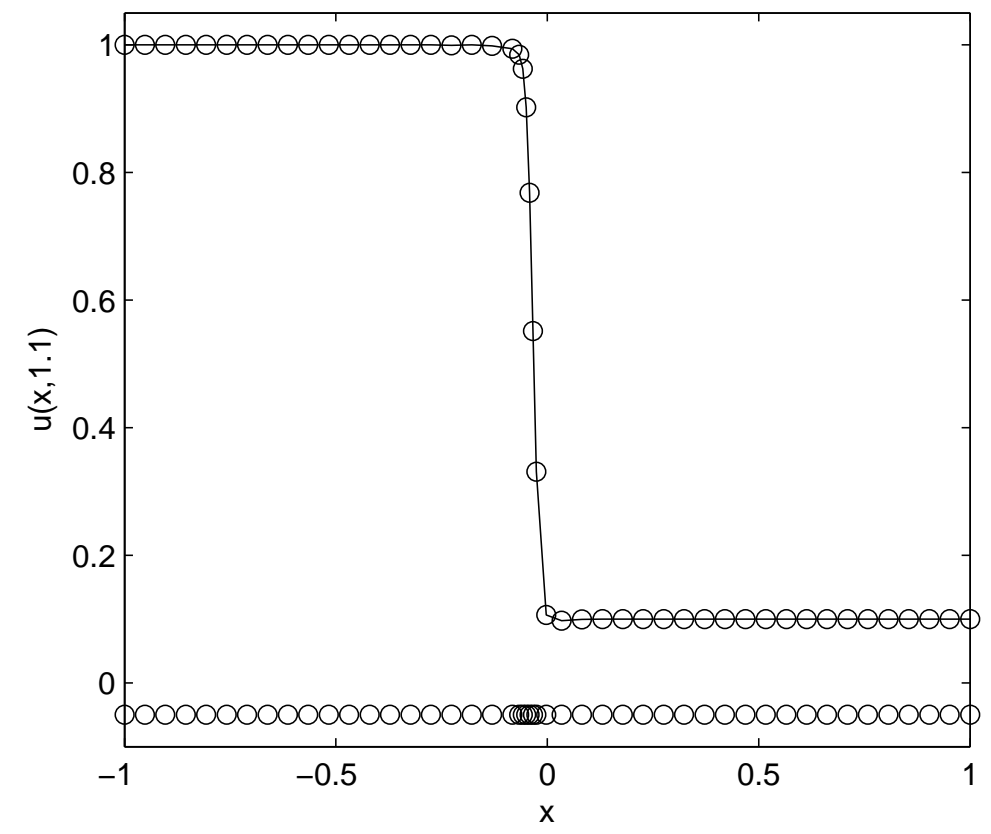

Fig. 3. Exact solution of Burgers' equation (solid) at $t=1.1$ from section 5.2. Open circles represent the center locations at time $t=1.1 \mathrm{using}$ the $\mathrm{MQ} \mathrm{RBF}$ and $N=47$. Results of the numerical experiment are given in table 4 .

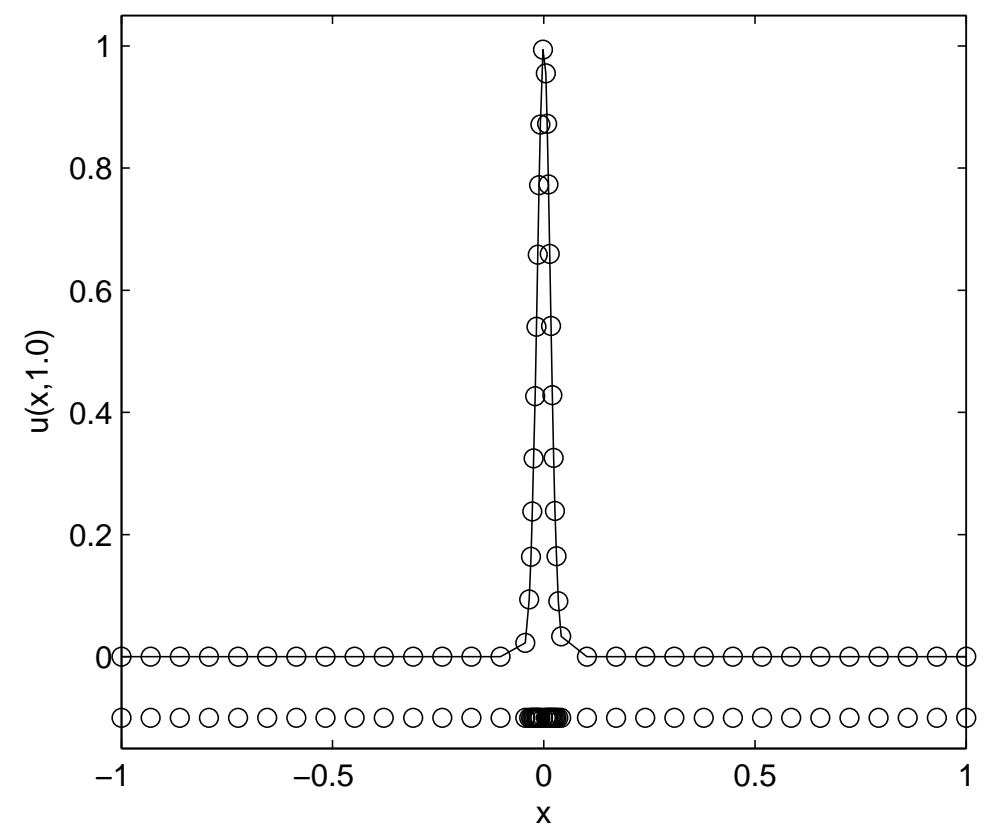

Fig. 4. Exact advection equation solution (solid) at $t=1$ from section 5.3. Open circles represent the center locations at time $t=1$ using the Wendland W42 RBF and $N=49$. Results of the numerical experiment are given in table 8 . 


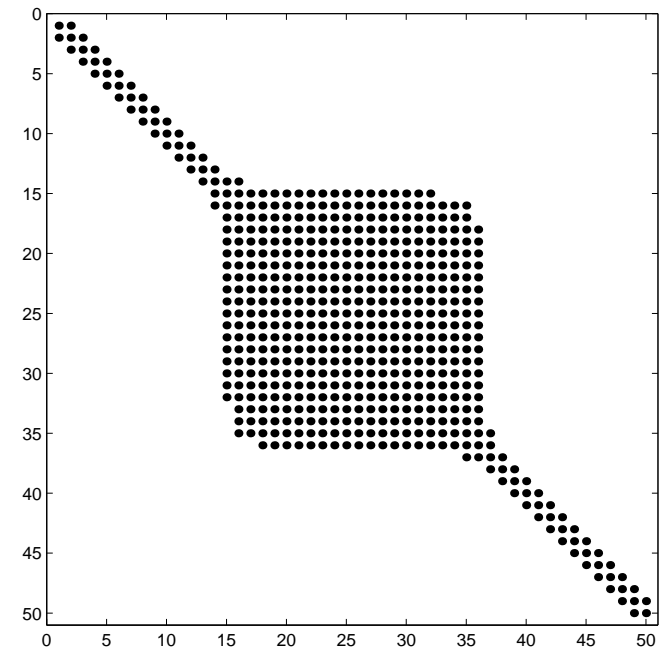

Fig. 5. Structure of the interpolation matrix $H$ at time $t=1$ with $N=49$ from the W42 numerical solution of the advection equation from section 5.3 and figure 4 . 